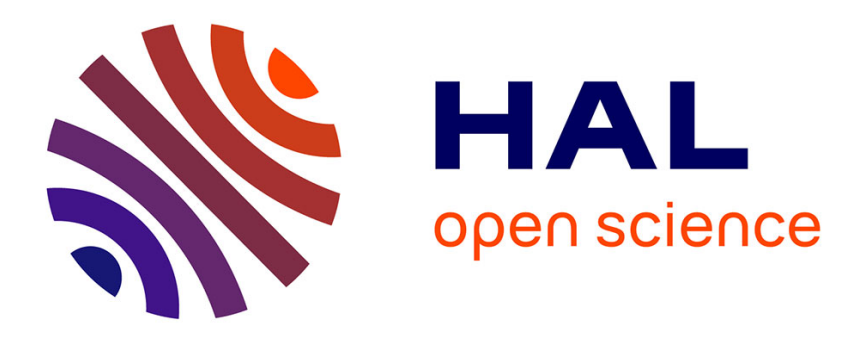

\title{
Picosecond and femtosecond Fourier pulse shape synthesis
}

\author{
A.M. Weiner, J.P. Heritage
}

\section{To cite this version:}

A.M. Weiner, J.P. Heritage. Picosecond and femtosecond Fourier pulse shape synthesis. Revue de Physique Appliquée, 1987, 22 (12), pp.1619-1628. 10.1051/rphysap:0198700220120161900 . jpa00245721

\section{HAL Id: jpa-00245721 https://hal.science/jpa-00245721}

Submitted on 1 Jan 1987

HAL is a multi-disciplinary open access archive for the deposit and dissemination of scientific research documents, whether they are published or not. The documents may come from teaching and research institutions in France or abroad, or from public or private research centers.
L'archive ouverte pluridisciplinaire HAL, est destinée au dépôt et à la diffusion de documents scientifiques de niveau recherche, publiés ou non, émanant des établissements d'enseignement et de recherche français ou étrangers, des laboratoires publics ou privés. 


\title{
Picosecond and femtosecond Fourier pulse shape synthesis
}

\author{
A. M. Weiner and J. P. Heritage \\ Bell Communications Research, 331 Newman Springs Road, Red Bank, NJ 07701-7020, U.S.A.
}

(Reçu le 19 juin 1987, accepté le 18 septembre 1987)

\begin{abstract}
Résumé. - Nous passons en revue la synthèse d'impulsions optiques cohérentes de forme arbitraire par filtrage spatial dans un compresseur d'impulsions à réseau. Nous décrivons des impulsions picosecondes mises en forme au moyen d'un compresseur d'impulsions à fibre et à réseau ainsi que des impulsions femtosecondes mises en forme au moyen d'un compresseur à réseau non dispersif. La discussion concerne la génération d'impulsions carrées cohérentes et d'impulsions « impaires » abruptement modulées en phase, des mesures par interférence temporelle à deux fentes du spectre de phase optique ainsi que l'encodage d'impulsions ultracourtes et le décodage par brouillage de phase. Nous concluons par une proposition d'interrupteur autodirectif optique ultrarapide basé sur le codage en phase dans le domaine des fréquences d'impulsions ultracourtes.

Abstract. - We review our work on the synthesis of arbitrarily shaped, coherent optical pulses, by spatial filtering in a grating pulse compressor. We describe picosecond pulses shaped using a fiber and grating pulse compressor and femtosecond pulses shaped using a nondispersive grating compressor. The discussion includes generation of coherent square pulses and abruptly phase-modulated « odd» pulses, two slit temporal interference measurements of the optical phase spectrum, and ultrashort pulse encryption and decoding by phase scrambling. We conclude with a proposal for an ultrafast optical self-routing switch based upon frequency domain phase coding of ultrashort pulses.
\end{abstract}

\section{Introduction.}

Due to continued progress in modelocked laser and pulse compression technology, optical pulses as short as six femtoseconds can now be generated [1]. In contrast to the advanced state of optical «pulse generators ", the development of optical " function generators » capable of generating arbitrarily shaped pulses has until recently received far less attention. In this paper we discuss a powerful new technique for picosecond and femtosecond pulse shape synthesis, which we illustrate by numerous experimental examples.

In the 1970's, Debois [2] and Agostinelli [3] demonstrated a technique for optical pulse shaping based on spatial filtering of optical frequency components which were physically dispersed by a pair of diffraction gratings. Due to the temporal dispersion of the gratings, however, the shaped pulses were necessarily chirped and stretched out in time compared to the input pulse. Our technique also utilizes spatial filtering within a grating compressor, but by eliminating or compensating the grating temporal dispersion, we obtain arbitrarily shaped coherent pulses which can be as short or even shorter than the starting pulse [4-7]. A review article by Froehly provides a comprehensive account of related pulse shaping work performed prior to 1983 [7].

We may describe our pulse shaping as time-domain Fourier optics, in analogy with spatial Fourier optics [8] used for image processing, etc ; this connection is also pointed out in [7]. In time-domain Fourier optics, a grating maps the optical frequency spectrum of an input pulse onto a filter plane ; in spatial Fourier optics, a lens takes the spatial Fourier transform of an object and places it at the filter plane. In both cases a spatially patterned mask is introduced at the filter plane in order to manipulate the Fourier spectrum. Adjustment of the spectrum results in either a shaped pulse (time domain) or a processed image (spatial domain). We anticipate that much of the knowledge and experience already accumulated in spatial Fourier optics will be relevent to time-domain Fourier optics as well.

The paper is structured as follows. In section 2, we discuss picosecond pulses shaped in a fiber and grating pulse compressor, including as examples square pulses, abruptly phase-modulated « odd» 
pulses and temporal interference measurements of the optical phase spectrum. In section 3 we enumerate the limitations of pulse shaping. In section 4 , we report high resolution femtosecond pulse shaping using a nondispersive grating compressor and describe experiments on pulse encryption and decoding. In section 5 we outline a proposal for an optical spread spectrum communications network based on pulse shaping; and in section 6 , we expand on the connection between pulse shaping and Fourier optics. In section 7 we summarize.

\section{Picosecond pulse shaping.}

2.1 EXPERIMENTAL APPARATUS. - The experimental apparatus for our picosecond pulse shaping experiments [4, 5] is a fiber and grating pulse compressor, as shown in figure 1 . Seventy-five picosecond, $1.06-\mu \mathrm{m}$ pulses from a cw mode-locked $\mathrm{Nd}$ : YAG laser are spectrally broadened in a $400-\mathrm{m}$ length of single-mode, polarization-preserving optical fiber and compressed to durations as short as 0.8 ps by using a double-pass grating pair [9]. After a single pass through the gratings, the optical spectral components are spatially dispersed. In this region a spectral window [10] is used to enhance the quality of the compressed pulse, and spatially patterned amplitude and phase masks are utilized to modify the pulse's Fourier spectrum. For maximum spectral resolution the fiber output spot is imaged onto the filter plane. A second pass through the grating pair reassembles the frequency components into a collimated beam, yielding a shaped compressed pulse which is the Fourier transform of the spatial amplitude and phase pattern transferred onto the frequency spectrum.

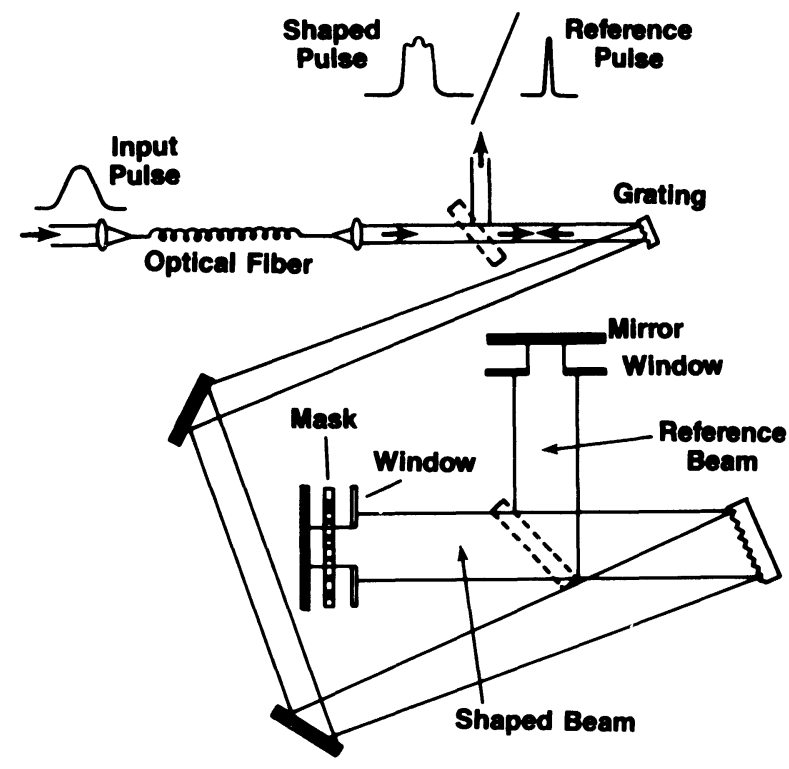

Fig. 1. - Experimental apparatus for picosecond pulse shaping experiments.
Pulse shape measurements are performed either by autocorrelation, or for more detail, by crosscorrelation. For the cross-correlation measurements, a beam splitter is placed in the compression apparatus after the first pass through the grating pair. The transmitted beam is windowed and shaped; the reflected beam is windowed only. Both beams are then directed back through the grating pair. In this way we obtain a short, subpicosecond reference pulse which is used to sample the longer and more complicated shaped pulse.

2.2 SPECTRAL WINDOWING. - Our first pulse shaping application was spectral windowing, a technique for enhancing the quality of compressed pulses [10]. It is well known that fiber group velocity dispersion (GVD) can linearize the chirp of self-phase-modulated pulses, resulting in efficient compression of the input pulse [11]. In the near infrared, however, GVD is small ; compression of $1.06-\mu \mathrm{m} \mathrm{Nd} \mathrm{:} \mathrm{YAG}$ lasers is often performed under conditions of negligible GVD, resulting in a nonlinear chirp and a compressed pulse with wings and sidelobes. Here we describe elimination of compressed pulse wings by spectral windowing [10]. An alternative technique for wing suppression based on nonlinear polarization rotation in the fiber is discussed in [12].

Spectral windowing is illustrated schematically in figure 2 , which shows the input pulse shape (a), and

a)

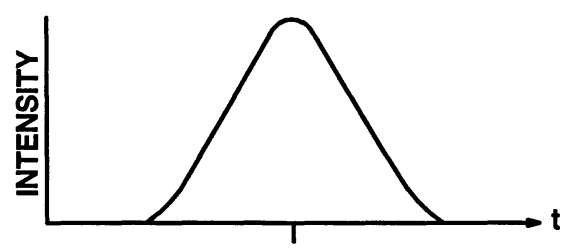

b)

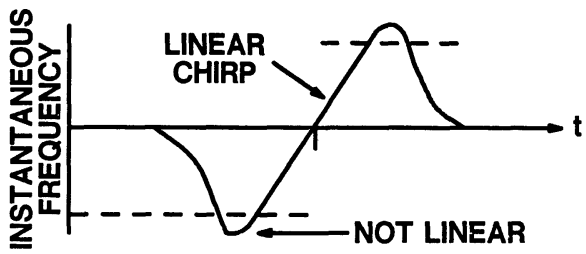

c)

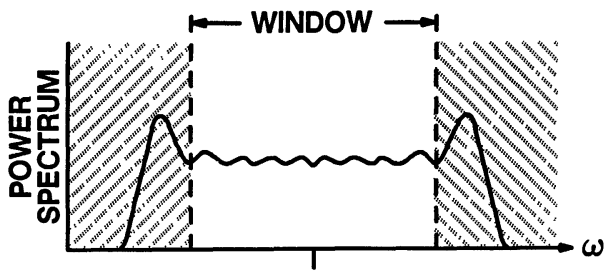

Fig. 2. - Suppression of compressed pulse wings by spectral windowing. a) Intensity profile of the input pulse. b) Chirped pulse instantaneous frequency profile, assuming no group velocity dispersion. c) Power spectrum of the chirped pulse. In $(b, c)$, the dashed lines indicate the position of a spectral window. 
the instantaneous frequency (b) and power spectrum (c) of the chirped pulse. For large frequency offsets the chirp is nonlinear and cannot be effectively compressed by the gratings. These offending frequency components can be eliminated using a spectral window positioned as indicated in figure $2 b, c$. The resultant wing clipping is demonstrated by figure $3 \mathrm{a}$, which shows autocorrelations of windowed and unwindowed pulses. Windowing results in dramatic wing reduction, with nearly zero pulse broadening. These results are emphasized by figure $3 b$, which shows windowed and unwindowed autocorrelations on an expanded scale. Suppression of the wings is accompanied by substantially reduced fluctuations, as observed using a real-time autocorrelator. These high quality windowed pulses are the starting point for the pulse shaping experiments described below.

2.3 COMPLEX SHAPED PULSES USING SIMPLE MASKS. - We previously reported several types of
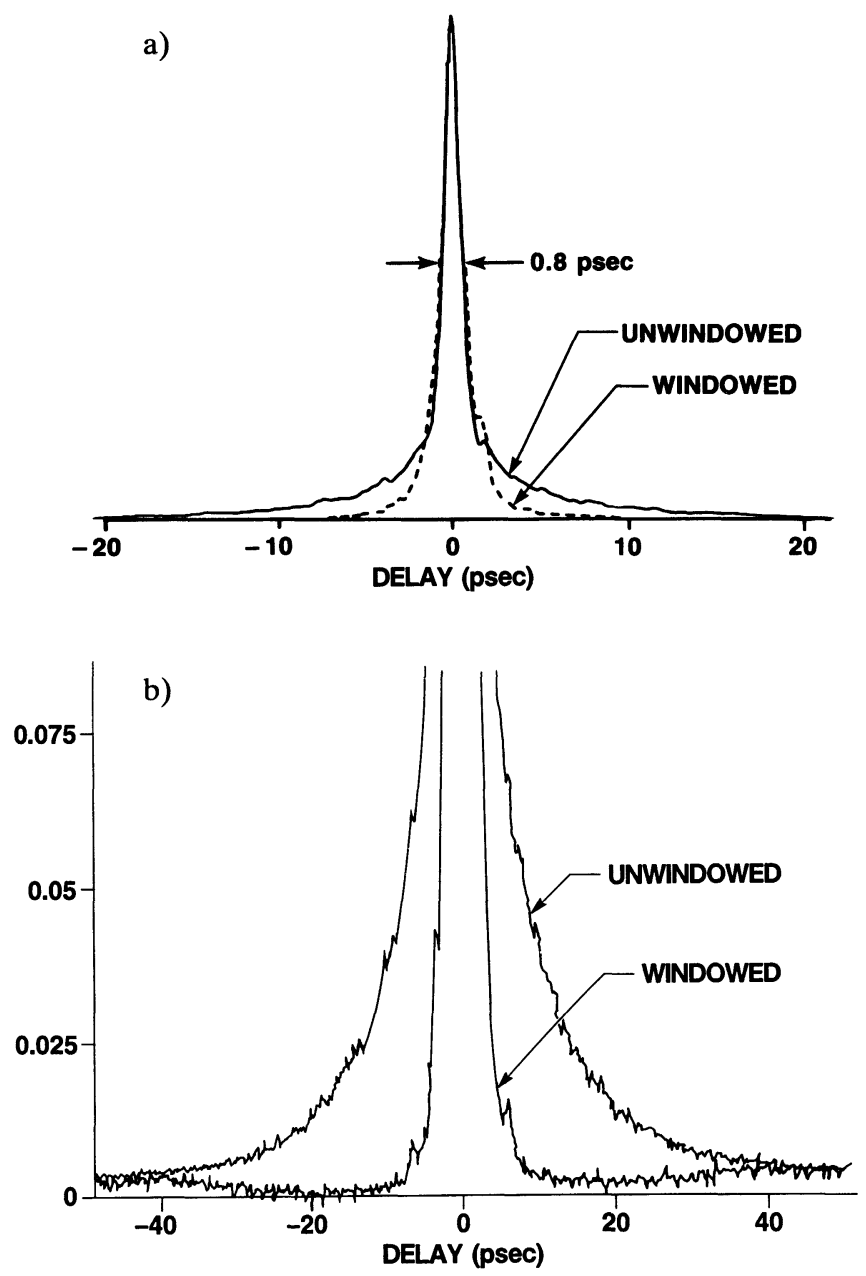

Fig. 3. - Autocorrelation measurements of windowed and unwindowed compressed pulses. a) The windowed pulse broadens only slightly, from 0.8 to $0.9 \mathrm{ps}$. b) The expanded vertical scale dramatizes the degree of wing suppression. shaped pulses produced using simple amplitude and phase masks [4]. For example, a spatially periodic interruption of the spectrum yields a sequence of pulses whose spacing in time is inversely proportional to the spatial period. We present in figure 4 measurements of such a Fourier transform pair : $4 \mathrm{a}$ is the periodically interrupted spectrum, and $4 \mathrm{~b}$ is the corresponding autocorrelation trace, which corresponds to a three pulse burst (five peaks in the autocorrelation). This measurement pair sensitively tests several properties of the Fourier transform. The pulse repetition period is the inverse of the spacing between adjacent spectral features, the width of individual pulses is determined by the total bandwith, and the breadth of the temporal envelope is controlled by the inverse width of the individual spectral features. In all these details the synthesized pulse train corresponds accurately to the Fourier transform of the shaped spectrum [6].
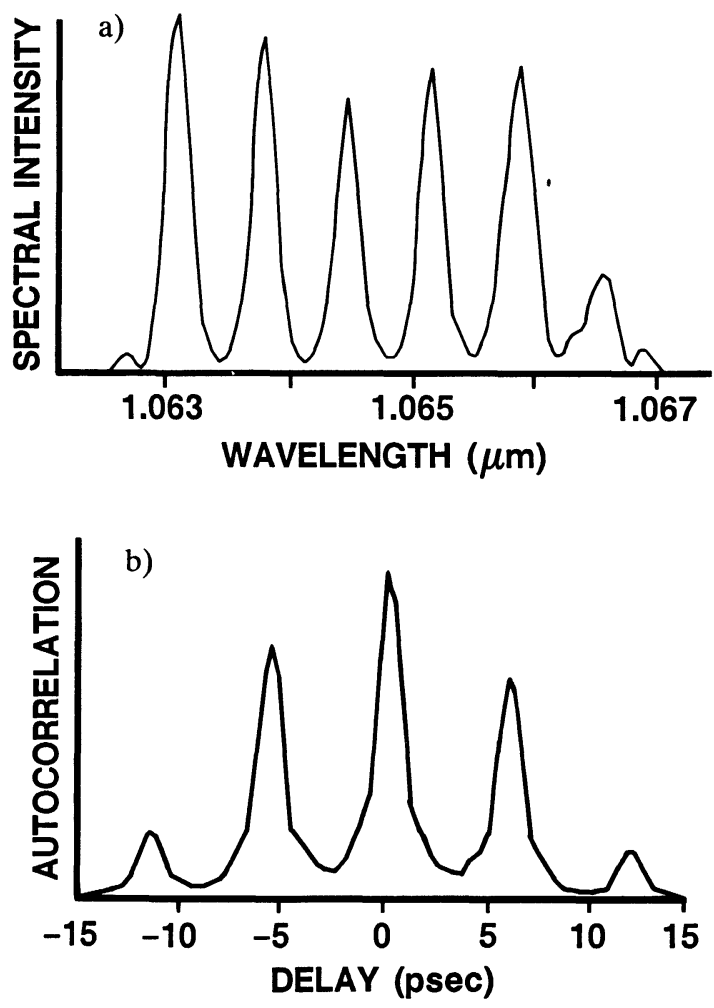

Fig. 4. - a) Periodically interrupted spectrum, and b) autocorrelation measurement of the resulting burst of pulses.

As a simple example of phase masking, we consider the « odd " pulse. When half of an originally symmetric spectrum undergoes a $\pi$ phase shift, it becomes an antisymmetric spectrum. The corresponding electric field will be an odd function of time ; the intensity profile will consist of two peaks separated by a null at $t=0$ where the phase of the carrier jumps abruptly by $\pi$. Such an odd pulse is closely 
related to the zero-area pulse produced by propagation through resonant atomic vapors [13].

The odd pulse was achieved experimentally by inserting a thin, transparent film into half of the spectrum. A thin pellicle, a microscope cover-glass slide and a thermally evaporated film of $\mathrm{SiO}$ covering half of a microscope slide all produced the same results when adjusted for $(2 n+1) \pi$ phase shift. A cross-correlation measurement of the odd pulse is shown in figure 5. As expected, the cross-correlation exhibits two equally intense pulses with a minimum at $t=0$.

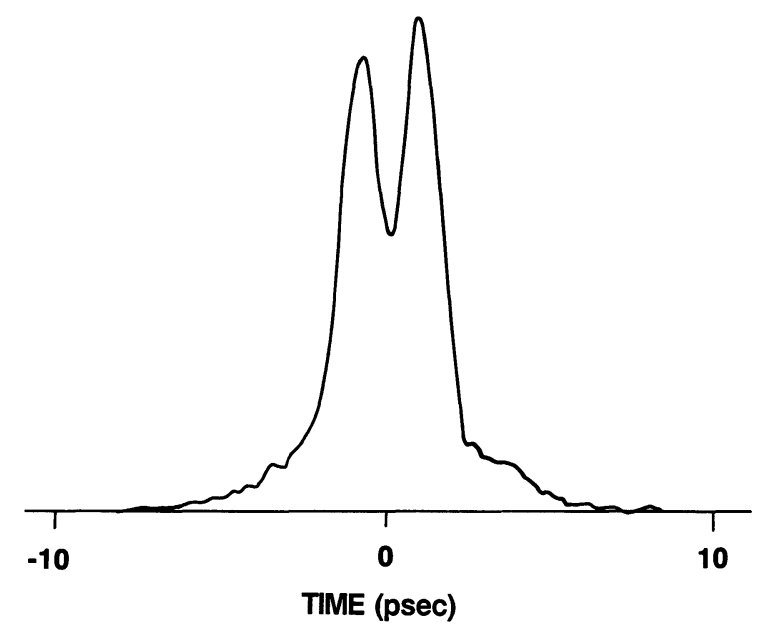

Fig. 5. - Cross-correlation measurement of a ps odd pulse.

2.4 THE SQUARE PULSE : A SIMPLE SHAPED PULSE USING A COMPLICATED MASK. - Above we described a variety of complicated pulse types which were generated using a rather simple set of spatial masks. In this section we discuss the synthesis of a phase-coherent, picosecond square pulse : a simple pulse shape that, however, corresponds to a rather complicated spectrum. The square pulse could potentially find application to nonlinear optical metrology and to future optical digital communication and signal-processing systems.

In order to generate a square pulse of duration $T$, it is necessary to produce a spectrum $E(\omega)$ shaped like a sinc function :

$$
E(\omega)=E_{0} T \frac{\sin (\Delta \omega T / 2)}{(\Delta \omega T / 2)} .
$$

Fabrication of a truncated $\sin (k x) / k x$ mask was described in [5]. The amplitude variation was implemented by a mask comprised of alternating opaque and transparent strips whose widths, always small compared with the spot size in order to maintain a smooth $E(\omega)$, were adjusted in order to produce the desired variation. The required $\pi$ phase shifts were obtained by etching a pattern of approp- riate depth on a second mask placed in series with the first.

A cross-correlation measurement of the square pulse is shown in figure 6a. The predicted crosscorrelation trace, calculated as described in [6] and shown in figure $6 \mathrm{~b}$, is in good agreement with the experimental results. The truncation of the spectrum owing to the finite available bandwidth $(3.3 \mathrm{~nm}$, $875 \mathrm{GHz}$ ) causes ripple on the square pulse and limits the $10-90 \%$ rise time to $0.9 \mathrm{ps}$. From the similarity of the measured and calculated pulse shapes, we conclude that the square pulse is very nearly transform limited and phase coherent.
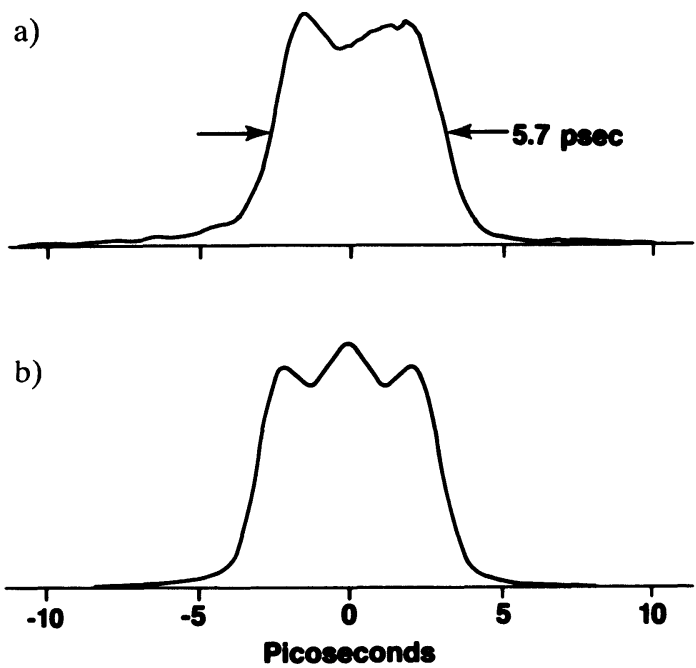

Fig. 6. - a) Cross-correlation measurement of ps square pulse. b) Predicted cross-correlation trace.

2.5 SPECTRAL PHASE MEASUREMENTS. - We have utilized the pulse shaping capabilities demonstrated in the preceding sections to perform novel two-slit temporal interference measurements of the optical phase spectrum of compressed pulses [14]. Our technique involves measuring the beat note between two optical frequency components selected by a pair of narrow slits placed at the filter plane. A related technique, introduced by Rothenburg [15], utilizes the beat note which arises when a chirped pulse propagates through a resonant atomic vapor. The principle of our phase measurement is illustrated by figure 7, which shows cross-correlation measurements of the resulting interference. When the two spectral components are in phase (top trace), the beat signal has a peak at $t=0$. When the two frequencies have a relative phase shift, the peaks of the interference pattern are shifted in time by a corresponding amount. In the lower trace, we have utilized a specially fabricated phase plate to deliberately introduce a $\pi$ phase shift between the two oscillators. The resulting shift in the temporal inter- 


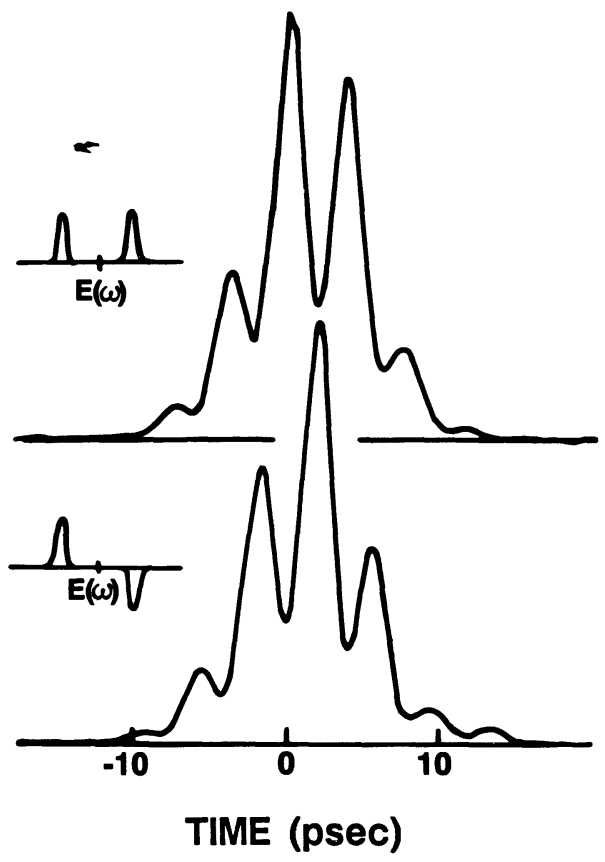

Fig. 7. - Upper trace : cross-correlation measurement of the temporal beat note between two isolated optical frequency components. Lower trace : cross-correlation of the shifted beat note which results when one of the frequencies is phase shifted by $\pi$. Alternatively, the shift in the temporal beat note can be used to measure the phase difference between two frequencies.

ference pattern is clearly evident and easily measurable.

We have applied this technique to investigate the phase spectrum of compressed pulses in the presence of intense stimulated Raman scattering (SRS) in a 145-meter fiber [14]. Under conditions of pulse walk-off [16], SRS preferentially depletes (steepens) the leading edge of the $1.06 \mu \mathrm{m}$ pump pulse, leading to a nonsymmetrically broadened self-phase-modulation spectrum which droops but extends further towards longer wavelengths [14, 16-18]. Our phase measurements indicate that the down-shifted frequency components of the pump pulse remain coherent and in phase out to the most extreme frequency shift. The up-shifted frequency components, however, maintain uniform phase only over a limited interval. At large frequency shifts, the phase of the up-shifted spectral components varies rapidly with frequency and loses coherence. These surprising results indicate that the phase spectrum is most uniform and most stable in the spectral region most affected by SRS.

Our spectral phase measurements indicate that high quality pulse compression should be possible even in the presence of intense SRS, by utilizing an asymmetric spectral window to select the linearly chirped, down-shifted spectral region. We have performed pulse compression experiments which confirm high quality compression under these condi- tions and which demonstrate in addition that compression of pulses clamped by extreme SRS is highly stabilized compared to conventional pulse compression. Stabilized compressed pulses as short as $0.6 \mathrm{ps}$ are obtained. A detailed discussion of pulse compression influenced by intense SRS is given elsewhere $[17,19]$.

\section{Limitations of pulse shaping.}

Although a wide and nearly arbitrary variety of pulse shapes may be synthesized by our spatial filtering technique, there are also limitations. These limitations, discussed in detail in [6], arise due to finite spectral resolution and finite available bandwidth. The shortest temporal feature $\delta t$ which can be present in the synthesized waveform is inversely related to the total bandwidth $B$, and the maximum duration $T$ of the temporal envelope is inversely related to the width $\delta f$ of the finest spectral feature - i.e.,

$$
T \simeq 1 / \delta f \text { and } \delta t \simeq 1 / B
$$

A useful measure of spectral resolution is given by the number of distinct spectral features which we can impose on a given bandwidth :

$$
m=B / \delta f .
$$

Since $B / \delta f=T / \delta t$, the number of independent temporal features which may be placed within a shaped pulse is roughly equal to $m$.

As discussed by [6], the minimum spectral width $\delta f$ depends on the spatial dispersion at the masking plane and on the finite spot size associated with any particular frequency component at the masking plane. These quantities are related in turn to the beam divergence of the input beam and to the angular dispersion of the grating. For gratings used at Littrow, $m$ can be as large as

$$
m=\frac{\Delta \lambda}{\lambda} \frac{\pi W}{d}
$$

where $\Delta \lambda$ is the pulse bandwidth in units of wavelength, $W$ is the beam radius of the input beam, and $d$ is the grating period. The spectral resolution improves as the bandwidth, the beam aperture and the number of grating lines per $\mathrm{mm}$ increase.

For the data shown in section $2, m$ was approximately 20 - i.e., we could manufacture pulse shapes as long as 20 psec, containing as many as 20 separate, 1-psec features. From a similar compressed Nd : YAG system, an optimized resolution $m \simeq 100$ is possible, using subpicosecond compressed pulses obtained under conditions of intense SRS [19]. Pulse shaping performed using femtosecond pulses permits further enhancement of resolution owing to the increased bandwidth. Using a $10 \mathrm{~nm}$ bandwidth, a 
$1 \mathrm{~cm}$ beam diameter and a 1800 line $/ \mathrm{mm}$ grating, $m>500$ should be possible. Thus, femtosecond pulse shaping will make possible synthesis of remarkably complex tailored pulses.

\section{Femtosecond pulse shaping.}

As an interesting and nontrivial extension of the picosecond pulse shaping work described in section 2 , we recently performed femtosecond pulse shaping experiments. These experiments required a modified, dispersion-free pulse shaping apparatus, which we will describe below. As a benefit of working in the femtosecond regime, it becomes possible to perform extremely high resolution pulse shaping. As an illustration of the complexity which can be designed into a pulse shape, we discuss frequency-domain phase-coding (or encryption) of fs pulses.

\subsection{NONDISPERSIVE GRATING COMPRESSOR. -} Our experiments utilize a balanced, colliding-pulsemode-locked (CPM) ring dye laser [20,21] as a source of $0.62-\mu \mathrm{m}$ wavelength, femtosecond pulses. Since these pulses are substantially bandwidth-limited (unchirped), a nondispersive grating apparatus is required. Such a nondispersive grating « compressor ", previously utilized by Froehly for picosecond pulse shaping experiments [7], is shown in figure 8. We use two 1700 line/mm gratings, separated by $60 \mathrm{~cm}$ and placed at the focal planes of a unit-magnification confocal lens pair. Pulse shaping is achieved by inserting spatially patterned amplitude and phase masks midway between the two lenses at the point where the optical spectral components experience maximal spatial separation. The shaped pulses are measured by cross-correlation, using fs pulses directly out of the CPM laser as the reference.

That the temporal dispersion normally introduced by gratings is eliminated by the lens pair may be understood as follows. The first lens performs a Fourier transform from the first grating to the

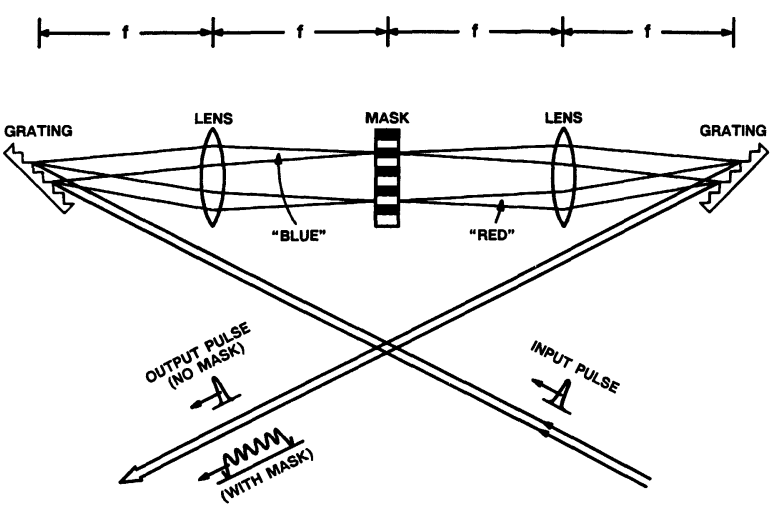

Fig. 8. - Nondispersive grating « compressor» apparatus. In our experiments the gratings are $1700 \mathrm{line} / \mathrm{mm}$, and the lens focal lengths are $f=150 \mathrm{~mm}$. midpoint between the lenses; the second lens performs a second Fourier transform from the midpoint to the second grating. Thus, propagation from grating to grating is described by two successive Fourier transformations; this is equivalent to a conventional grating pair [22] with zero separation between gratings !

The lens pair removes temporal dispersion to a remarkable degree : we have performed autocorrelation measurements of 74 fs pulses directly out of the CPM laser and after passing through the nondispersive grating apparatus, and observe broadening by only $2 \mathrm{fs}$, to $76 \mathrm{fs}$. A conventional grating pair without the lenses (with the same $60 \mathrm{~cm}$ separation) would broaden the pulse by nearly three orders of magnitude (to $\approx 50 \mathrm{ps}$ ).

As an aside, we note that similar grating and lens arrangements can be utilized to produce either positive or negative dispersion, by moving the gratings either closer to or further from the lenses, respectively. The generalized grating compressor so obtained was studied theoretically by Martinez [23]. Experimentally, we have used such a compressor in the negative dispersion region to achieve subpicosecond compression and high resolution shaping $(m \approx 80)$ of $1.06-\mu \mathrm{m}, \mathrm{Nd}$ : YAG laser pulses [19, see also Sect. 2].

4.2 FEMTOSECOND PULSE TRAINS AND FEMTOSECOND ODD PULSES. - We first demonstrate femtosecond pulse shaping by producing simple pulse shapes which were previously produced in the picosecond regime. Figure 9 shows a cross-correlation measurement of a fs odd pulse generated using a total spectral width of $7.0 \mathrm{~nm}(5.5 \mathrm{THz})$. Except for the shorter time scale, the odd pulse is nearly identical with that produced in the picosecond fiber and grating compressor (Fig. 5). Evidently the pulse shaping capabilities developed first with picosecond pulses can be directly transferred to the femtosecond regime.

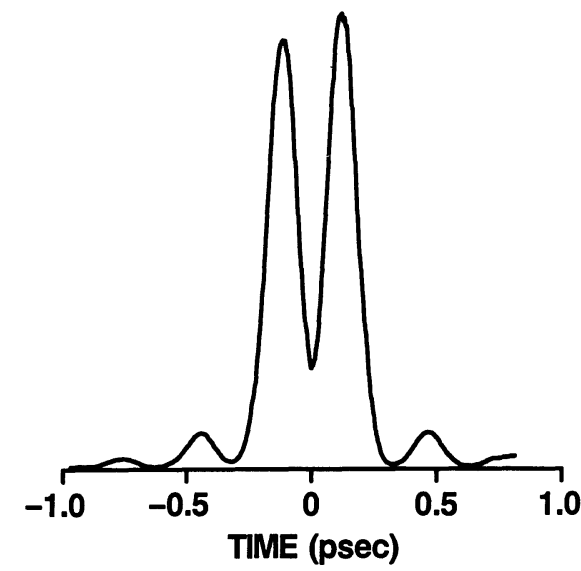

Fig. 9. - Cross-correlation measurement of a fs odd pulse. A 75 fs pulse directly from the CPM laser is used as the reference pulse for the cross-correlation. 
Another simple example illustrates the high resolution pulse shaping achievable with femtosecond pulses. An amplitude mask selects two isolated spectral components, separated by $3.37 \mathrm{~nm}$ $(2.6 \mathrm{THz})$. As previously, the two frequencies interfere, producing the high frequency tone burst shown in figure 10a. Compared to the picosecond examples (Figs. 4b and 7),there is now a remarkable amount of detail. The fs cross-correlation trace shows twenty distinct temporal peaks, with an estimated order of magnitude increase in complexity possible. By introducing a phase mask to phase shift one frequency component by $\pi$, we can interchange the position of the peaks and nulls (Fig. 10b), again analogous to the previous picosecond experiments. The $2.6 \mathrm{Thz}$ beat frequency, corresponding to a period of only $380 \mathrm{fs}$, is an order of magnitude faster than the highest frequency tone bursts previously generated [24].
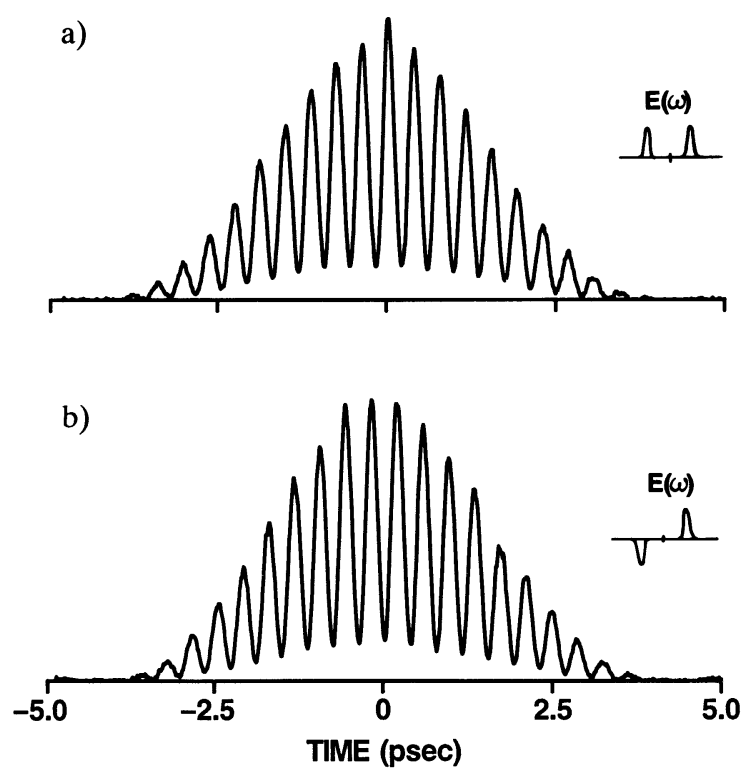

Fig. 10. - a) Cross-correlation measurement of a $2.6 \mathrm{THz}$ burst of femtosecond pulses. b) Shifted burst of pulses resulting when one frequency is shifted by $\pi$.

\subsection{ENCRYPTION AND DECODING OF FEMTOSECOND} PULSES. - As a further example of the exquisite control attainable by high resolution pulse shaping, we discuss frequency-domain phase-coding of femtosecond pulses. We show that a pseudorandom phase mask can scramble the phases of the spectral components which comprise the ultrashort pulse, thus transforming an intense fs pulse into a low level, picosecond duration pseudonoise burst. We will also show that the original femtosecond pulse can be reconstituted from such a pseudonoise burst. Finally, in section 5 , we will propose that such encryption and decoding of ultrashort pulses could form the basis of a picosecond, code-division multiplexed, optical communications network.

A note on terminology : by "encryption " we mean encoding; secrecy is not implied.

An example of spectral phase coding is shown in figure 11. A 44-element, pseudorandom binary phase mask, shown in the inset, is used to scramble the spectral phases. A cross-correlation measurement of the complicated pseudonoise burst which results is plotted in figure 11a. This "encrypted" waveform is spread over more than 5 ps and contains close to fifty identifiable features. The theoretical intensity profile, calculated with no adjustable parameters, is plotted in figure 11b. The good agreement between theory and experiment emphasizes the excellent precision available with our technique.
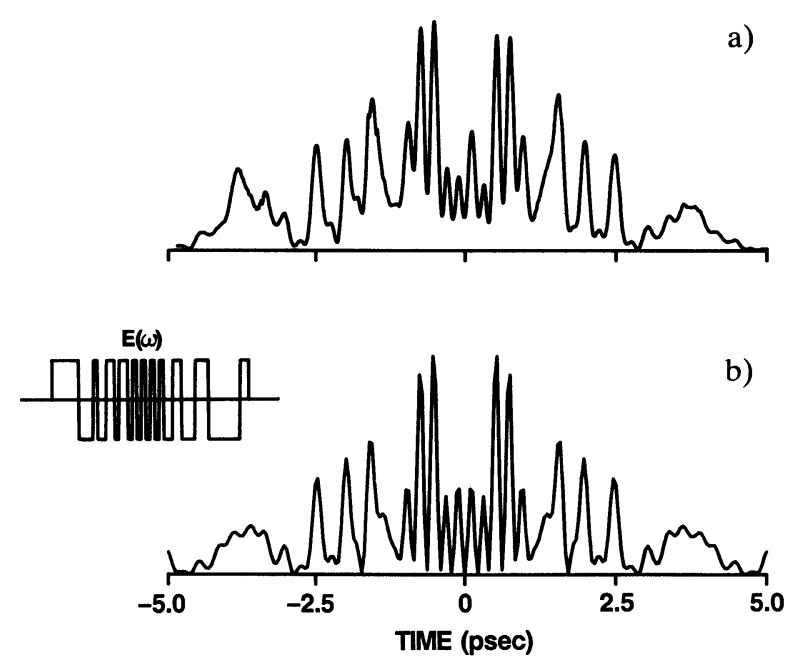

Fig. 11. - Frequency-domain phase-coding of fs pulses. a) Cross-correlation measurement of the phase coded waveform. The phase code is shown in the inset. b) Calculated intensity profile of the phase coded waveform.

Autocorrelation measurements of uncoded, coded and decoded pulses are shown in figure 12. This series of autocorrelation measurements lays the foundation for the proposed picosecond optical network outlined in the following section. Figure 12a depicts the autocorrelation of the incident, uncoded pulses together with that of the coded, or encrypted, pulses. The contrast ratio of $\approx 25: 1$ illustrates the dramatic reduction in intensity which accompanies encryption. The autocorrelation of the coded pulse is shown on an expanded vertical scale in figure $12 \mathrm{~b}$. The clearly visible coherence spike is further proof that have succeeded in willfully transforming a coherent femtosecond pulse into a noise burst!

In order to reconstitute the original femtosecond pulse, we place a second, phase conjugate mask into the pulse shaping apparatus. This phase conjugate mask decodes (or unscrambles) the spectral phases scrambled by the first mask, thus restoring the initial 

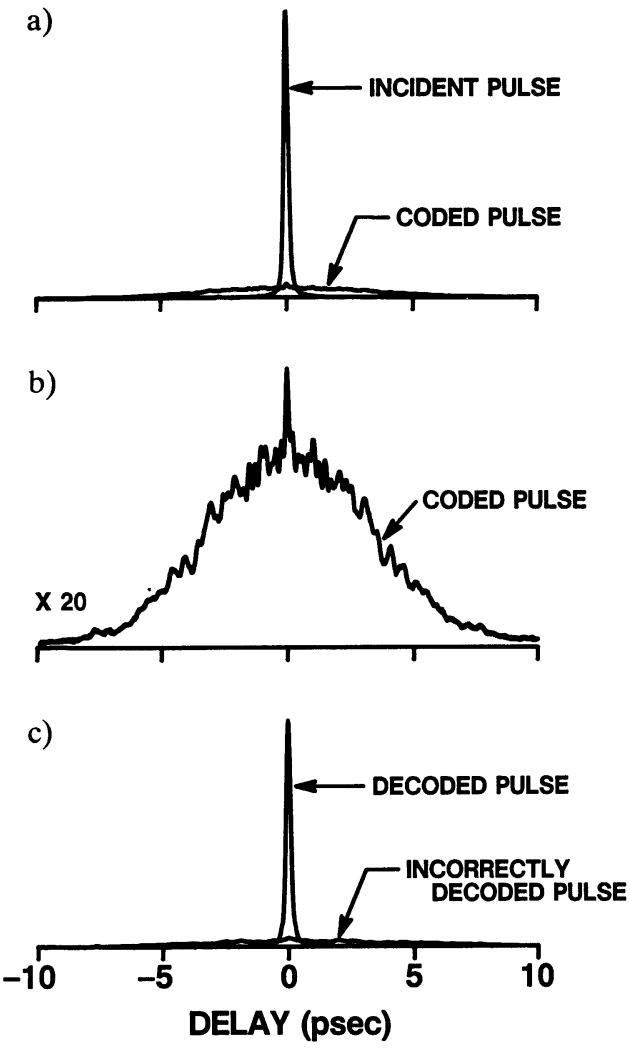

Fig. 12. - Autocorrelation measurements of uncoded, coded and decoded pulses. a) Uncoded and coded pulses ; b) Coded pulse, vertical scale expanded $20 \times$; c) Correctly and incorrectly decoded pulses.

pulse. The autocorrelation of a reconstituted femtosecond pulse is shown in figure $12 \mathrm{c}$; even on an expanded scale, there is no broadening compared to the original pulse. On the other hand, if the second mask is not phase conjugate to the first, the spectral phases are rearranged but not unscrambled. In that case the waveform remains a spread, low intensity pseudonoise burst.

\section{A picosecond optical self-routing switch.}

We propose that frequency-domain phase-coding of individual ultrashort light pulses, as described in the previous section, could form the basis for an ultrahigh-speed, optical spread spectrum communications network. Specifically, we propose that a fiberbased code-division multiple-access (CDMA) system could be implemented by spectral encryption and decoding of ultrashort pulses. Other CDMA systems have traditionally been based upon time-domain encryption and decoding and have been restricted to the lower bit rates attainable using direct laser modulation and electronic processing. A general discussion of CDMA can be found in the spread spectrum literature [25]; optical implementations are discussed in [26, 27].

A schematic diagram of the proposed CDMA network is shown by figure $13 . N$ subscriber stations are connected via a passive $N \times N$ directional coupler. Each transmitter station is equipped with a picosecond or femtosecond source and a multielement phase modulator (the encoder) ; each receiver is equipped with a similar phase modulator (the decoder) and an optical threshold device. Each receiving station is assigned a unique «lock», or access code, which it imposes on its phase decoder. In order for transmitter $i$ to communicate with receiver $j$, $i$ must encode using a «key » which is phase conjugate to lock $\mathrm{j}$. In that case, decoder $\mathrm{j}$ will
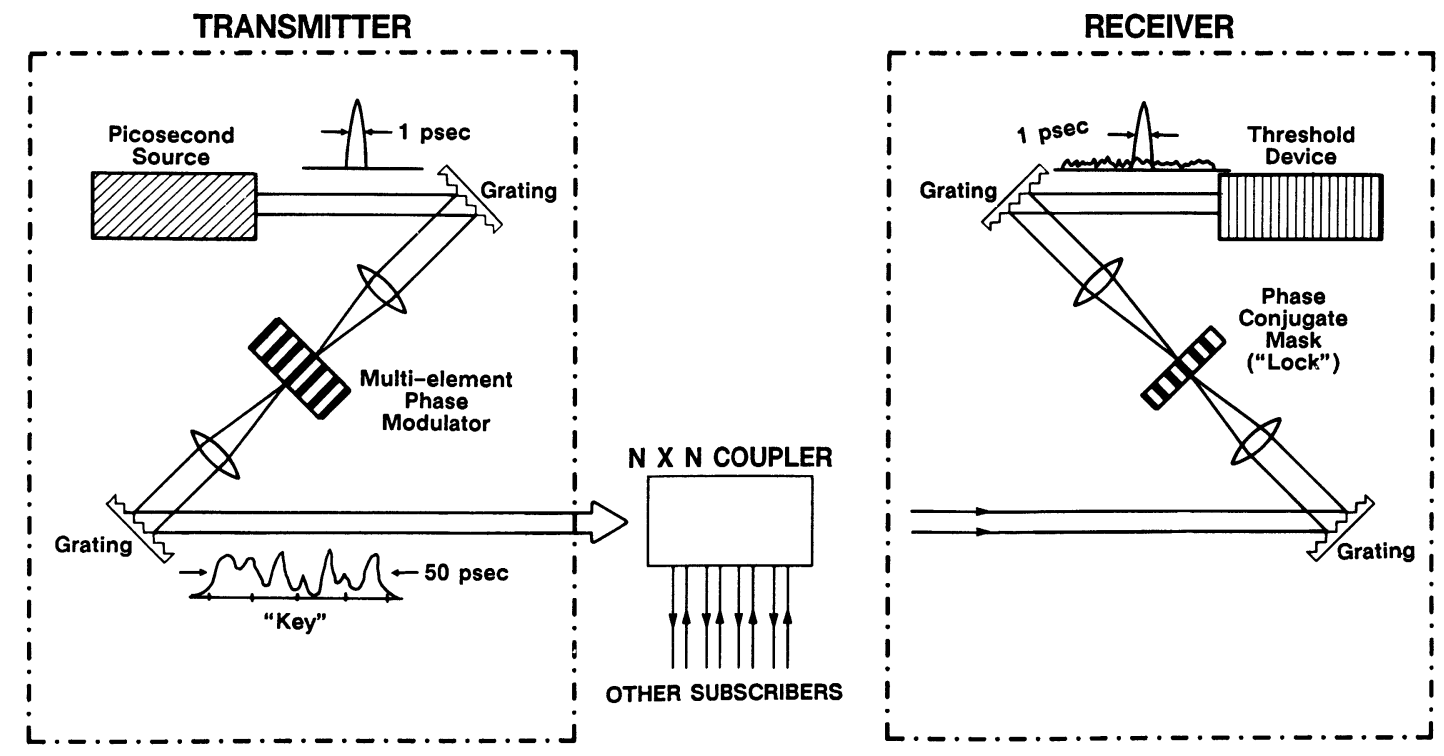

Fig. 13. - An optical self-routing switch : proposal for an ultrashort pulse code-division multiple-access communications network. For purposes of clarity, the grating pairs are drawn with artistic license ; the correct orientation of the gratings is shown in figure 8 . 
reassemble the signal transmitted from $i$ into an intense bandwidth-limited pulse which can be detected by the thresholder. A signal transmitted from some other station $\mathrm{k}$, whose key does not fit the lock

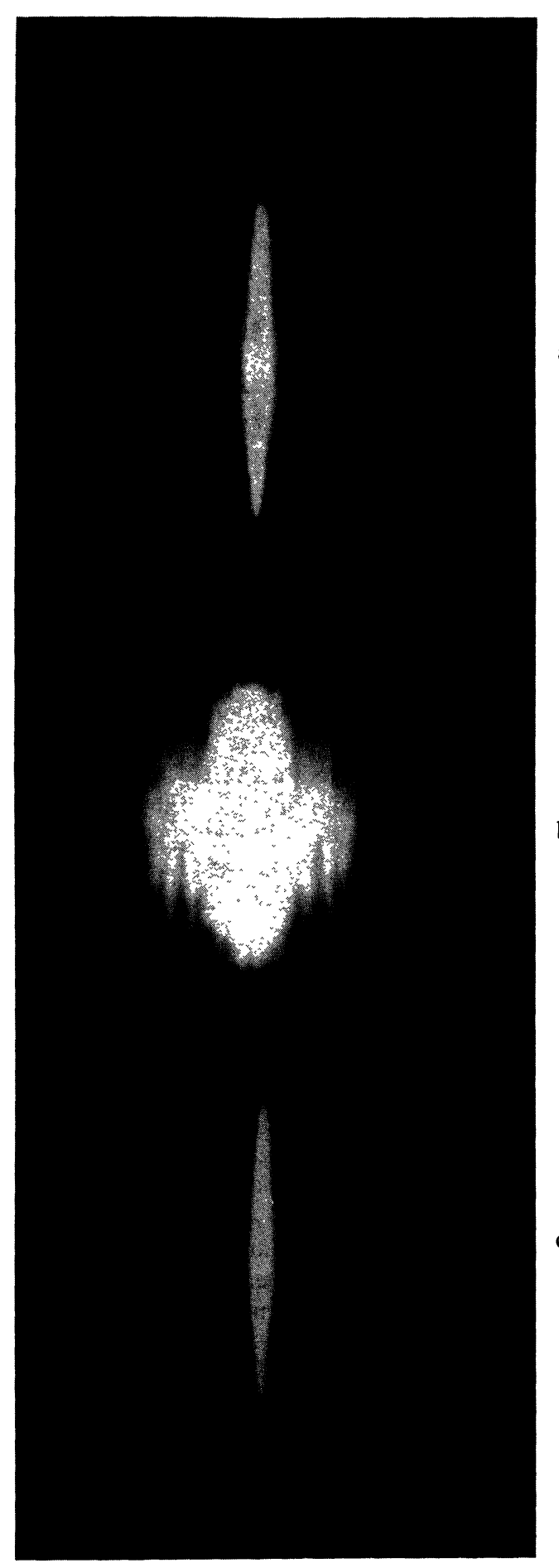

a)

b)

c)

Fig. 14. - Far-field diffraction pattern from the phase mask used for femtosecond pulse phase coding. The diffraction pattern corresponds to the intensity profile of masked pulses. Some details are partially washed out due to the low dynamic range of the color film. a) The unmasked «pulse». b) The phase-coded pulse. c) The decoded pulse. at $\mathrm{j}$, will remain a low intensity noise burst and will be rejected by the thresholder.

The advantages conferred by such a system would be several. Multiple pairs of subscribers could communicate simultaneously and asynchronously through the same communication channel (either free-space or fiber optic), without interference. Free-space channel capacities in the several hundred gigabit range could be achieved, e.g., by multiplexing several hundred Gbit coded data streams, without requiring picosecond modulators or electronics. Furthermore, a transmitter could change its code (and the destination for the transmitted data) on a bit by bit basis, still without picosecond modulators. Essentially this system is an optical self-routing switch : transmitted data includes on it the information required to direct itself to the attention of the intended receiver alone.

\section{Time-domain Fourier optics.}

In the introduction we pointed out the analogy between pulse shaping (« time-domain Fourier optics ») and spatial Fourier optics. Here we emphasize that connection by noting that free-space diffraction performs a spatial Fourier transform. The far-field diffraction pattern of a laser beam transmitted through a spatially patterned mask is the Fourier transform of that pattern squared; the pulse shape which results when that same mask is placed at the filter plane of a grating compressor is the same squared Fourier transform. Thus, free-space diffraction may be used to preview the results of pulse shaping experiments.

Figure 14 shows an example. A He-Ne laser beam diffracts off the pseudorandom phase mask used for encrypting fs pulses (see Fig. 11). Figures 14a and $14 \mathrm{~b}$, respectively, represent the original unmasked pulse and the masked pulse, which is a low intensity, longer duration pseudonoise burst. The details evident in figure $14 \mathrm{~b}$ are directly identifiable with the temporal features present on the measured waveform (Fig. 11). Finally, figure 14c depicts a decoded pulse, in which a second phase mask has been used to cancel the phase changes caused by the first, thus reconstituting the original femtosecond pulse. The similarity between figure 14 and figure 12 is striking.

\section{Summary.}

In summary, we have demonstrated the ability to shape ultrashort pulses according to specification, by spatially filtering optical frequency components dispersed within a grating pulse compressor. By using a special dispersion-free grating apparatus, we have generated highly complex optical waveforms with femtosecond control. Synthesis of waveforms con- 
sisting of five hundred individually programmable femtosecond features should be possible. As an example, we have demonstrated encryption and decoding of ultrashort pulses and have suggested that this technology could be utilized for a codedivision multiple-access optical communications network. We anticipate that the prefabricated masks used in the current work could be replaced by high speed multielement modulators, allowing real-time pulse shape synthesis and encoding. Fourier trans- form pulse shaping is the time domain analogue to spatial Fourier optics and should provide functionalities in the ultrafast time domain which Fourier optics currently achieves in the spatial domain, such as information processing and pattern recognition. Applications to spread spectrum communications and to multiple-pulse excitation, coherent timedomain spectroscopy should also be possible.

We gratefully acknowledge E. M. Kirschner for fabrication of masks used in our experiments.

\section{References}

[1] Brito-Cruz, C. H., Fork, R. L. and Shank, C. V., Compression of optical pulses to 6 fs using cubic phase distortion compensation, Conference on Lasers and Electro-optics, Baltimore, MD, April, 1987.

[2] Desbois, J., Gires, F. and Tournois, P., IEEE J. Quantum Electron. QE-9 (1973) 213.

[3] Agostinelli, J., Harvey, G., Stone, T. and Gabel, C., Appl. Opt. 18 (1979) 2500.

[4] Heritage, J. P., Weiner, A. M. and Thurston, R. N., Opt. Lett. 10 (1985) 609.

[5] Weiner, A. M., Heritage, J. P. and Thurston, R. N., Opt. Lett. 11 (1986) 153.

[6] Thurston, R. N., Heritage, J. P., Weiner, A. M. and TOMLINSON, W. J., IEEE J. Quantum Electron. QE-22 (1986) 682.

[7] Froehly, C., Colombeau, B. and Vampouille, M., in Progress in Optics $X X$, Ed E. Wolf (North-Holland, Amsterdam) 1983, pp. 65-153.

[8] Goodman, J. W., Introduction to Fourier Optics (McGraw-Hill, San Francisco) 1968.

[9] Johnson, A. M., Stolen, R. H. and Simpson, W. M., Appl. Phys. Lett. 44 (1984) 729.

[10] Heritage, J. P., Thurston, R. N., Tomlinson, W. J., Weiner, A. M., and Stolen, R. H., Appl. Phys. Lett. 47 (1985) 87.

[11] Grischkowsky, D. and Balant, A. C., Appl. Phys. Lett. 41 (1983) 1.

[12] Halas, N. J. and Grischkowsky, D., Appl. Phys. Lett. 48 (1986) 823.

[13] Rothenberg, J. E., GrischKowsky, D. and Balant, A. C., Phys. Rev. Lett. 53 (1984) 552.

[14] Heritage, J. P., Weiner, A. M. and Thurston, R. N., in Ultrafast Phenomena $V$, Eds G. R.
Fleming and A. E. Siegman (Springer, Berlin), 1986, pp. 33-37.

[15] Rothenberg, J. E. and GrischKowsky, D. R., $J$. Opt. Soc. Am. B 2 (1985) 626.

[16] Stolen, R. H. and Johnson, A. M., IEEE J. Quantum Electron. QE-22 (1986) 2154.

[17] Weiner, A. M., Heritage, J. P. and Stolen, R. H., submitted to J. Opt. Soc. Am. B.

[18] ZYSSET, B. and WEBER, H. P., Temporal and spectral investigation of $N d: Y A G$ pulse propagation in optical fibers and its application to pulse compression, Conference on Lasers and Electro-optics, San Francisco, CA, June 1986.

[19] Heritage, J. P., Weiner, A. M. and Martinez, O. E., submitted to IEEE J. Quantum Electron.

[20] Fork, R. L., Greene, B. I. and Shank, C. V., Appl. Phys. Lett. 38 (1981) 671.

[21] VAldmanis, J. A., ForK, R. L. and Gordon, J. P., Opt. Lett. 10 (1985) 131.

[22] Treacy, E. B., IEEE J. Quantum Electron. QE-5 (1969) 454.

[23] Martinez, O. E., IEEE J. Quantum Electron. QE23 (1987) 59.

[24] TAI, K., Tomita, A., Jewell J. L. and Hasegawa, A., Appl. Phys. Lett. 49 (1986) 236.

[25] SKaug, R., Spread Spectrum in Communications (Peter Peregrinus) 1985.

[26] Salehi, J. A. and Brackett, C. A., Fundamental Principles of Fiber Optics Code-Division Multiple-Access, IEEE International Conference on Communications, Seattle, WA, June 1987.

[27] Prucnal, P. R., Santoro, M. A. and Fan, T. R., IEEE J. Lightwave Technol. LT-4 (1986) 547. 